\title{
Perfil da mortalidade por intoxicação com medicamentos no Brasil, 1996-2005: retrato de uma década
}

\author{
Profile of mortality by intoxication with medication \\ in Brazil, 1996-2005: portrait of a decade
}

Daniel Marques Mota ${ }^{1}$

José Romério Rabelo Melo ${ }^{1}$

Daniel Roberto Coradi de Freitas ${ }^{1}$

Márcio Machado ${ }^{2}$

${ }^{1}$ Agência Nacional de Vigilância Sanitária (Anvisa). SIA Trecho 5 Quadra Especial 57, Lote 200. Bloco D $1^{\circ}$ andar, Sala NUVIG. 71.205-050 Brasília DF daniel.mota@anvisa.gov.br ${ }^{2}$ Pharmacy Administration, University of Toronto

\begin{abstract}
The occurrence of deaths caused by intoxication with medication have been considered a worsening public health problem. The study describes the epidemiological profile of medicationrelated intoxication in the general Brazilian population from 1996 to 2005. A descriptive study was conducted with mortality data obtained from the Mortality Information System of the Brazilian Ministry of Health. Deaths were selected according to the codes of the International Classification of Diseases (ICD-10). A total of 4,403 deaths were found inn males (53.9\%), bachelors (53.7\%) and the 20 to 39 year-old age bracket (44\%). The majority of deaths were caused by intentional selfintoxication using anticonvulsants, sedatives, antiparkinsonians and psychotropics. The standardized mortality rate was higher in the Midwest region and Potential Life-Years Lost increased by 15.5\%. The study showed the characteristics and variations in mortality by intoxication with medication in Brazil, which can be a reflex of the medication consumption patterns of the country, indicating the need for enhancement of sanitary vigilance policies.
\end{abstract}

Key words Adverse effects, Pharmacoepidemiology, Intoxication, Medication, Mortality
Resumo A ocorrência de óbitos por intoxicação com medicamentos tem sido considerada um dos agravos de saúde pública. O estudo descreve o perfil epidemiológico da mortalidade por intoxicação com medicamentos na população do Brasil entre 1996 e 2005. Realizou-se um estudo descritivo com dados do Sistema de Informações sobre Mortalidade do Ministério da Saúde. Selecionaram-se os óbitos segundo a Classificação Internacional de Doenças (CID-10). Foram identificados 4.403 óbitos ocorridos em homens (53,9\%), solteiros (53,7\%) e faixa etária de 20-39 anos (44,0\%). A maioria dos óbitos foi por autointoxicação intencional por anticonvulsivantes, sedativos, antiparkinsonianos e psicotrópicos. A taxa padronizada de mortalidade foi maior na região Centro-Oeste e os Anos Potenciais de Vida Perdidos aumentou durante o período estudado em 15,50\%. O estudo apresentou as características e variações na mortalidade por intoxicação com medicamentos no Brasil que pode ser um reflexo do padrão de consumo dos medicamentos no país atrelado à necessidade de aprimoramento das políticas de vigilância sanitária.

Palavras-chave Efeitos adversos, Farmacoepidemiologia, Intoxicação, Medicamentos, Mortalidade 


\section{Introdução}

Medicamento pode ser definido como produto farmacêutico, tecnicamente obtido ou elaborado, com finalidade profilática, curativa, paliativa ou para fins de diagnóstico ${ }^{1}$, mas que pode ser um potencial causador de dano e óbito. Neste sentido, vários autores citam o medicamento como um dos principais agentes responsáveis por intoxicações resultando em atendimento, nas emergências toxicológicas, hospitalização e óbitos em diferentes países ${ }^{2-11}$. Apesar disso, autoridades sanitárias de países em desenvolvimento ainda não se sensibilizaram efetivamente para a vigilância desse agravo.

No Brasil, chama a atenção a necessidade de aprimoramento da regulação da publicidade, facilidade na aquisição de medicamentos sob prescrição médica, inexistência de legislação específica sobre embalagens seguras, escassas iniciativas de desenvolvimento da atenção farmacêutica e o padrão do consumo de medicamentos pela população, caracterizado pela automedicação, polifarmácia, uso indevido e indiscriminado de antibióticos e psicotrópicos ${ }^{12-14}$. Estes aspectos contribuem para o aumento dos casos e óbitos por intoxicação com medicamentos.

Entre 1986 e 2006, foram registrados no Brasil, pelo Sistema Nacional de Informações Tóxico-Farmacológicas (SINITOX), 1.220.987 casos de intoxicação, com um total de 7.597 (0,6\%) óbitos. A partir de 1994, os medicamentos assumiram a primeira posição no conjunto de agentes tóxicos estudados, respondendo por $24,5 \%$ dos casos de intoxicação registrados no país. Ao longo dos vinte anos de registro, os medicamentos foram responsáveis por 1.327 óbitos, resultando em uma taxa de letalidade de $0,4 \%{ }^{15}$.

Dados do Sistema de Informações Hospitalares do Ministério da Saúde relacionados com hospitais do Estado do Rio de Janeiro apontam a intoxicação como o segundo tipo mais comum entre os agravos provocados por medicamentos, com 924 (27\%) casos, entre 1999 e 2002. Do total das intoxicações, $56(6,1 \%)$ pacientes evoluíram para o óbito ${ }^{16}$.

No Brasil, há uma carência de estudos sobre a mortalidade por intoxicação com medicamentos, em nível nacional, baseados exclusivamente em dados de estatísticas vitais ${ }^{17}$. Os estudos publicados têm utilizado como fonte de dados, basicamente, os registros disponíveis pelos SINITOX e centros estaduais/municipais de informações e assistência toxicológicas (CIAT) ${ }^{3-4,12,18-19}$. Esses estudos estão focados em espaços regiona- lizados e os dados do SINITOX apresentam lacunas que comprometem a qualidade e a quantidade das informações sobre esse agravo como: i) número de CIAT (oito Unidades da Federação não dispõem de centros) que enviam os dados ao SINITOX é insuficiente para cobrir toda a extensão territorial do país; ii) notificação realizada de maneira espontânea, ocorrendo, na maioria das vezes, com o objetivo de obter informação sobre o manejo e onde buscar atendimento de saúde; iii) falta de padronização dos dados entre os diversos CIAT; e iv) grande parte dos casos de intoxicação é atendida diretamente na rede de serviços de saúde, sem que os centros tomem conhecimento sobre o fato ${ }^{3,15,19}$.

A análise de dados secundários disponíveis no Sistema de Informações sobre Mortalidade do Ministério da Saúde (SIM/MS), com abrangência nacional e qualidade aceitável ${ }^{20-21}$, pode ser outra fonte de dados em potencial sobre mortalidade por intoxicação com medicamentos no Brasil. Este sistema de informação, alimentado pela ficha de Declaração de Óbito, é composto por 44 variáveis e gerenciado pela Secretaria de Vigilância em Saúde do Ministério da Saúde em conjunto com as Secretarias Estaduais e Municipais de Saúde. Neste contexto, o objetivo deste artigo é descrever o perfil epidemiológico da mortalidade por intoxicação com medicamentos, na população brasileira entre $1^{\circ}$ de janeiro de 1996 e 31 de dezembro de 2005, a partir de uma análise dos dados registrados no SIM/MS.

\section{Metodologia}

Realizou-se um estudo descritivo cuja população do estudo foi constituída pelo universo dos óbitos por intoxicação com medicamentos registrados no SIM/MS

Os dez bancos de dados anuais foram coletados, em novembro de 2008 no sítio eletrônico do Departamento de Informática do Ministério da Saúde (DATASUS) ${ }^{22}$, os quais disponibilizam informações individuais por paciente falecido. Foi analisada a distribuição dos óbitos por sexo, idade, estado civil, anos de estudos, raça/cor, ocupação, circunstância e local de ocorrência do óbito, bem como por região de residência e causa básica do óbito codificada pela Classificação Internacional de Doenças (CID-10) da Organização Mundial da Saúde em sua décima revisão ${ }^{23}$.

Neste estudo, foi considerado como caso de "óbito por intoxicação com medicamentos" todo registro de paciente notificado no SIM/MS que 
apresentou como causa básica de óbito códigos da CID-10 associados à intoxicação com medicamentos. Os demais agravos provocados por medicamentos, como erros de medicação e reação adversa, foram excluídos do estudo. Para a identificação dos óbitos foi realizada uma revisão dos códigos da CID-10 e os selecionados estão apresentados no Quadro 1.

$\mathrm{Na}$ construção das taxas de mortalidade por 1.000.000 habitantes foi utilizada a população para cada ano, fornecida pelo Instituto Brasileiro de Geografia e Estatística e obtida em sítio eletrônico ${ }^{24}$. A taxa de mortalidade proporcional por ano foi calculada como sendo o número de óbitos por intoxicação com medicamentos dividido pelo total de óbitos por todas as causas e multiplicado por 100. Para a análise por regiões, foram calculadas as taxas padronizadas de mortalidade pelo método direto ${ }^{25}$, sendo tomada como padrão a distribuição etária da população brasileira do censo do ano de 2000 .

O desfecho Anos Potenciais de Vida Perdidos (APVP) foi calculado pela soma das diferenças entre a expectativa média de vida no Brasil, que neste estudo foi considerada como 70 anos, e as idades em que ocorreu o óbito de pessoas víti-

Quadro 1. Campos diagnósticos selecionados da CID-10 para identificação dos óbitos por intoxicação com medicamentos. Brasil, 1996-2005.

\begin{tabular}{|c|c|}
\hline Código & Capítulo/Descrição \\
\hline \multicolumn{2}{|r|}{ Capitulo: $V$ - Transtornos mentais e comportamentais } \\
\hline $\begin{array}{l}\text { F11.0 } \\
\text { F13.0 } \\
\text { F16.0 } \\
\text { F19.0 }\end{array}$ & $\begin{array}{l}\text { Intoxicação aguda por uso de opióides } \\
\text { Intoxicação aguda por uso de sedativos ou hipnóticos } \\
\text { Intoxicação aguda por uso de alucinógenos } \\
\text { Intoxicação aguda por uso de múltiplos fármacos e uso de outras substancias } \\
\text { psicoativas }\end{array}$ \\
\hline \multicolumn{2}{|r|}{ Capitulo: XVI - Algumas afecções originadas no período perinatal } \\
\hline P93 & Reações e intoxicações devido a fármacos administrados ao feto e neonato \\
\hline \multicolumn{2}{|c|}{ Capitulo: XIX - Lesões, envenenamento e algumas outras consequências de causas externas } \\
\hline T36 (T36.0 - T36.9) & Intoxicação por antibióticos sistêmicos \\
\hline T37 (T37.0 - T37.9) & Intoxicação por outros anti-infectantes e antiparasitários \\
\hline T38 (T38.0 - T38.9) & Intoxicação por hormônios e seus substitutos sintéticos e antagonistas \\
\hline T39 (T39.0 - T39.9) & Intoxicação por analgésicos não opiáceos, antipiréticos e antireumáticos \\
\hline $\mathrm{T} 40.2$ & Intoxicação por codeína e morfina \\
\hline T40.3 & Intoxicação por metadona \\
\hline T40.4 & Intoxicação por petidina \\
\hline T41 (T41.0 - T41.5) & Intoxicação por anestésicos e gases terapêuticos \\
\hline T42 (T42.0 - T42.8) & $\begin{array}{l}\text { Intoxicação por antiepilépticos e fármacos sedativo-hipnóticos e } \\
\text { antiparkinsonianos }\end{array}$ \\
\hline T43 (T43.0 - T41.9) & Intoxicação por fármacos antipsicóticos \\
\hline T44 (T44.0 - T44.9) & $\begin{array}{l}\text { Intoxicação por fármacos que primariamente afetam o sistema nervoso } \\
\text { autônomo }\end{array}$ \\
\hline T45 (T45.0 - T45.9) & Intoxicação por agentes primariamente sistêmicos e hematológicos \\
\hline T46 (T46.0 - T46.9) & Intoxicação por agentes que primariamente afetam o sistema cardiovascular \\
\hline T47 (T47.0 - T47.9) & Intoxicação por agentes que primariamente afetam o sistema gastrointestinal \\
\hline T48 (T48.0 - T48.9) & $\begin{array}{l}\text { Intoxicação por agentes que atuam primariamente em músculos lisos e esqueléticos } \\
\text { e no sistema respiratório }\end{array}$ \\
\hline T49 (T49.0 - T49.9) & $\begin{array}{l}\text { Intoxicação por agentes que primariamente afetam a pele e membrana mucosa } \\
\text { e fármacos oftalmológicos, otorrinolaringológicos e odontológicos }\end{array}$ \\
\hline T50 (T50.0 - T50.5) & $\begin{array}{l}\text { Intoxicação por diuréticos e outros fármacos não especificados, medicamentos } \\
\text { e substâncias biológicas }\end{array}$ \\
\hline T96 & Sequela de intoxicação por fármacos, medicamentos e substâncias biológicas \\
\hline
\end{tabular}


mas de intoxicação com medicamentos e por todas as causas. A idade limite de 70 anos foi baseada no valor mediano das expectativas de vida para o país entre 1996 e 2005. Os registros das pessoas que morreram com 70 anos ou mais foram desconsiderados para o cálculo da estimativa dos APVP. A média de APVP por óbito para cada ano foi calculada a partir da divisão do total de APVP pelo número de óbitos.

Para o cálculo da taxa de variação anual média (TVAM) foi utilizada a seguinte fórmula matemática: TVAM $(\%)=(n \vee$ valor final/valor inicial) - 1 x 100, onde " $n$ " representa o número de anos estudados.

A análise dos dados foi realizada nos software Epinfo versão 6.04d e Tabwin 3.2, sendo que foram excluídos os campos não preenchidos das bases de dados. Tabelas univariadas foram utilizadas para descrever o perfil da mortalidade a partir da frequência das variáveis estudadas. O teste qui-quadrado foi utilizado para compara- ção de proporções ao nível de 5\% de significância estatística. O projeto foi aprovado pela Comissão Nacional de Ética em Pesquisa (CONEP).

\section{Resultados}

No período estudado (1996 a 2005), dos 9.588.501 óbitos registrados no SIM/MS foram identificados $4.403(0,04 \%)$ relacionados à intoxicação com medicamentos, equivalentes à frequência de 4,6 óbitos/10.000 registros. Os óbitos ocorreram, sobretudo, em homens (53,9\%), raça/cor branca $(53,8 \%)$, indivíduos solteiros $(53,7 \%)$ e com 4 a 7 anos de estudo (27,8\%). A informação sobre raça/ cor disponível no SIM/MS no ano de 1996 apresentou $98 \%$ do total de registros não preenchidos, enquanto que para a variável "anos de estudos", entre 1996 e 1999, as opções de respostas foram diferentes quando comparadas as do período posterior e utilizada neste estudo (Tabela 1).

Quadro 1. continuação

\begin{tabular}{|l|l|}
\hline Código & \multicolumn{1}{c|}{ Capítulo/Descrição } \\
\hline \multicolumn{1}{|c|}{ Capitulo: XX - Causas externas de morbidade e de mortalidade } \\
\hline X40 (X40.0 -X40.9) & $\begin{array}{l}\text { Intoxicação acidental causada por exposição a analgésicos não opiáceos, } \\
\text { antipiréticos e antireumáticos } \\
\text { Intoxicação acidental causada por exposição a antiepilépticos, sedativos- } \\
\text { hipnóticos, fármacos antiparkinsonianos e psicotrópicos }\end{array}$ \\
X41 (X41.0 -X41.9) & $\begin{array}{l}\text { Intoxicação acidental causada por exposição a narcóticos e alucinógenos } \\
\text { Intoxicação acidental causada por exposição a outros fármacos que atuam no } \\
\text { sistemanervoso autônomo }\end{array}$ \\
X43 (X43.0 -X42.9 -X43.9) & $\begin{array}{l}\text { Intoxicação acidental causada por exposição a outros fármacos não } \\
\text { especificados, medicamentos e substancias biológicas }\end{array}$ \\
Intoxicação intencional causada por exposição a analgésicos não opiáceos, \\
antipiréticos e antireumáticos
\end{tabular}

*Evento de intenção não determinada. 
A principal circunstância do óbito por intoxicação com medicamentos foi o suicídio $(57,2 \%)$, sendo que em mais de $34 \%$ dos registros esta variável não era informada. Em relação à ocupação dos adultos, o maior percentual registrado foi para prendas domésticas $(23,5 \%)$. Dentre as regiões, as maiores proporções de óbitos foram observadas no Sudeste $(42,6 \%)$ e Nordeste $(23,3 \%)$. Para as variáveis estudadas, a comparação de suas proporções com o grupo de referência (R) apresentou diferenças estatisticamente significativas $(p<0,0001$ e $p<0,01)$, a exceção da variável "anos de estudo" ( $\mathrm{p}>0,05)$ (Tabela 1$)$.

O número de óbitos causados por intoxicação com medicamentos entre 1996 e 2005 foram, respectivamente, 438 e 516, enquanto que o número total de óbitos por todas as causas para os mesmos anos foi de 908.883 e 988.151. Comparando o ano de 1996 com 2005, os óbitos por intoxicação com medicamentos e por todas as causas apresentaram, respectivamente, uma taxa de crescimento de $17,8 \%$ e $8,7 \%$. Ao longo da década, a TVAM da mortalidade por intoxicação com medicamentos para o Brasil foi de 1,84\% e para todas as causas foi registrado um valor de $0,93 \%$. Na população do país essa taxa ficou em $1,78 \%$. A análise dos dados de mortalidade proporcional revelou que os anos de 1998 e 2000 apresentaram a maior $(0,06 \%)$ e a menor taxa $(0,03 \%)$, respectivamente.

A taxa bruta de mortalidade por intoxicação com medicamentos para o Brasil variou de 2,01 (2000) a 3,23 (1998) por 1.000.000 habitantes, sendo que no último ano analisado (2005) esta taxa resultou em 2,81/1.000.000 habitantes (Figura 1). Em 1996, a região Nordeste registrou a menor taxa padronizada de mortalidade por intoxicação com medicamento (TMIMp) $(1,67 /$ 1.000.000 habitantes), enquanto o Centro-Oeste obteve o maior valor com 3,66/1.000.000 habitantes. A partir de 2002, o Centro-Oeste passou a apresentar, de forma constante, valores superiores às taxas observadas para as outras regiões do país. No último ano estudado, esta região permaneceu com a maior taxa de mortalidade (5,05/1.000.000 habitantes), enquanto para a Norte foi registrado o menor valor $(1,31 /$ 1.000.000 habitantes).

Aproximadamente 99\% dos óbitos por intoxicação com medicamentos pertenceram ao capítulo XX da CID-10 - causas externas de morbidade e mortalidade, sendo que nenhum registro foi classificado no capítulo XIX que aborda sobre lesões, envenenamento e algumas outras consequências de causas externas. As outras cau-
Tabela 1. Caracterização da mortalidade por intoxicação com medicamentos, segundo variáveis selecionadas. Brasil, 1996-2005 $(\mathrm{N}=4.403)$.

\begin{tabular}{|c|c|c|c|}
\hline Variável & $\mathbf{N}$ & $\%$ & p-valor \\
\hline \multicolumn{4}{|l|}{ Sexo } \\
\hline Masculino (R) & 2.372 & 53,8 & \multirow[t]{2}{*}{$<0,0001$} \\
\hline Feminino & 2.030 & 46,1 & \\
\hline \multicolumn{4}{|l|}{ Raça/cor $(\mathrm{N}=3.251)$} \\
\hline Branca (R) & 1.749 & 53,8 & \multirow[t]{2}{*}{$<0,0001$} \\
\hline Parda & 1.211 & 37,2 & \\
\hline \multicolumn{4}{|l|}{ Estado civil $(\mathrm{N}=4.220)$} \\
\hline Solteiro (R) & 2.267 & 53,7 & \multirow[t]{2}{*}{$<0,0001$} \\
\hline Casado & 1.308 & 31,0 & \\
\hline \multicolumn{4}{|l|}{ Anos de estudo $(\mathrm{N}=1.951)$} \\
\hline 4 a $7(\mathrm{R})$ & 543 & 27,8 & \multirow[t]{3}{*}{$\mathrm{p}>0,05$} \\
\hline 1 a 3 & 447 & 22,9 & \\
\hline Nenhuma & 458 & 23,5 & \\
\hline \multicolumn{4}{|l|}{ Ocupação $(\mathrm{N}=3.772)$} \\
\hline Prendas domésticas (R) & 887 & 23,5 & \multirow[t]{5}{*}{$<0,0001$} \\
\hline Estudante & 391 & 10,4 & \\
\hline Empregador & 376 & 10,0 & \\
\hline Trabalhador volante na agricultura & 344 & 9,1 & \\
\hline Horticultor & 245 & 6,5 & \\
\hline \multicolumn{4}{|l|}{ Circunstância do óbito $(\mathrm{N}=3.693)$} \\
\hline Suicídio (R) & 2.111 & 57,2 & \multirow[t]{3}{*}{$<0,0001$} \\
\hline Ignorado & 1.527 & 34,7 & \\
\hline Acidental & 709 & 19,2 & \\
\hline \multicolumn{4}{|l|}{$\begin{array}{l}\text { Local de ocorrência do óbito } \\
(\mathrm{N}=4.392)\end{array}$} \\
\hline Hospital (R) & 3.167 & 72,1 & \multirow[t]{2}{*}{$<0,0001$} \\
\hline Domicílio & 764 & 17,4 & \\
\hline \multicolumn{4}{|l|}{ Região de residência } \\
\hline Sul (R) & 776 & 17,6 & \multirow[t]{5}{*}{$<0,01$} \\
\hline Norte & 289 & 6,6 & \\
\hline Nordeste & 1.026 & 23,3 & \\
\hline Sudeste & 1.876 & 42,6 & \\
\hline Centro-Oeste & 436 & 9,9 & \\
\hline
\end{tabular}

$\mathrm{R}=$ grupo de referência.

sas básicas de óbitos envolveram os capítulos $\mathrm{V}$ - transtornos mentais e comportamentais $(1,0 \%)$ e XVI - Algumas afecções originadas no período perinatal $(0,2 \%)$. Entre as causas de óbitos cujas classes de medicamentos foram identificadas, a maior proporção de mortes registrada foi por autointoxicação intencional por anticonvulsivantes, sedativos, antiparkinsonianos e psicotrópicos $(15,2 \%)$, considerando o valor total de óbitos por intoxicação com medicamentos.

A Tabela 2 apresenta os cinco principais códigos da CID-10, relacionados à intoxicação com medicamentos mais frequentes para os anos de 1996 e 2005. A quantidade de códigos da CID-10 em 1996 correspondeu a $15,0 \%$ do total selecionado para o estudo, sendo que em 2005 esse dado foi maior $(18,7 \%)$. Em termos qualitativos, não 


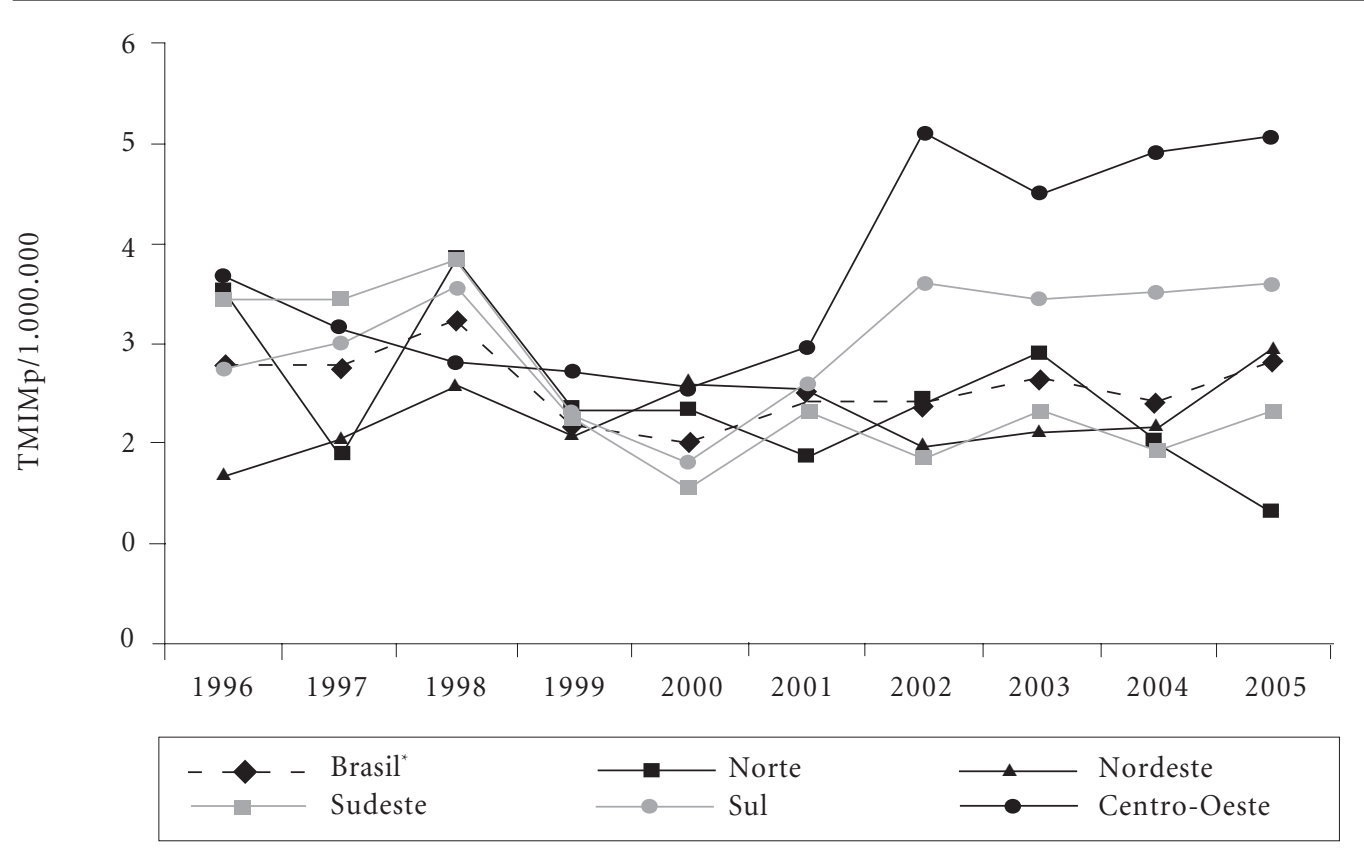

Figura 1. Evolução das taxas padronizadas de mortalidade por intoxicação com medicamentos (TMIMp/ 1.000.000), segundo Regiões do Brasil, 1996-2005 ( $\mathrm{N}=4.403)$.

Tabela 2. Comparação das cinco principais causas básicas de óbito para os anos de 1996 e 2005. Brasil, 1996-2005.

\begin{tabular}{|c|c|c|c|}
\hline Ano & Código/Descrição (Quantidade total de CID-10 = 326) & $\mathbf{N}$ & $\%$ \\
\hline \multirow{6}{*}{$\begin{array}{c}1996 \\
(\mathrm{~N}=438)\end{array}$} & Quantidade de códigos da CID-10 $=49(15,0 \%)$ & & \\
\hline & $\begin{array}{l}\text { Y14.9 - Intoxicação por exposição a outras drogas, medicamentos e } \\
\text { substâncias biológicas e as não especificadas, intenção não determinada - } \\
\text { local não especificado }\end{array}$ & 135 & $30,8^{*}$ \\
\hline & $\begin{array}{l}\text { X64.9 - Autointoxicação por exposição, intencional, a outras drogas, } \\
\text { medicamentos e substâncias biológicas e às não especificadas - local não } \\
\text { especificado }\end{array}$ & 55 & $12,6^{* *}$ \\
\hline & $\begin{array}{l}\text { X44.9 - Intoxicação acidental por exposição a outras drogas, medicamentos e } \\
\text { substâncias biológicas não especificadas - local não especificado }\end{array}$ & 37 & 8,4 \\
\hline & $\begin{array}{l}\text { X64.0 - Autointoxicação por exposição, intencional, a outras drogas, } \\
\text { medicamentos e substâncias biológicas e às não especificadas - residência }\end{array}$ & 35 & $8,0^{* *}$ \\
\hline & $\begin{array}{l}\text { X61.9 - Autointoxicação por exposição, intencional, a drogas } \\
\text { anticonvulsivantes [antiepilépticos], sedativos, hipnóticos, } \\
\text { antiparkinsonianos e psicotrópicos não classificados em outra parte - local } \\
\text { não especificado }\end{array}$ & 30 & $6,8^{* *}$ \\
\hline \multirow{6}{*}{$\begin{array}{c}2005 \\
(\mathrm{~N}=516)\end{array}$} & Quantidade de códigos da CID-10 = $61(18,7 \%)$ & & \\
\hline & $\begin{array}{l}\text { Y14.9 - Intoxicação por exposição a outras drogas, medicamentos e } \\
\text { substâncias biológicas e as não especificadas, intenção não determinada - } \\
\text { local não especificado }\end{array}$ & 82 & $15,9^{*}$ \\
\hline & $\begin{array}{l}\text { X64.9 - Autointoxicação por exposição, intencional, a outras drogas, } \\
\text { medicamentos e substâncias biológicas e às não especificadas - local não } \\
\text { especificado }\end{array}$ & 81 & $15,7^{\star *}$ \\
\hline & $\begin{array}{l}\text { X61.9 - Autointoxicação por exposição, intencional, a drogas } \\
\text { anticonvulsivantes [antiepilépticos], sedativos, hipnóticos, } \\
\text { antiparkinsonianos e psicotrópicos não classificados em outra parte - local } \\
\text { não especificado }\end{array}$ & 55 & $10,7^{\star *}$ \\
\hline & $\begin{array}{l}\text { X64.0 - Autointoxicação por exposição, intencional, a outras drogas, } \\
\text { medicamentos e substâncias biológicas e às não especificadas - residência }\end{array}$ & 41 & $7,9^{* *}$ \\
\hline & $\begin{array}{l}\text { X61.0 - Intoxicação intencional causada por exposição a antiepilépticos, } \\
\text { sedativos-hipnóticos, fármacos antiparkinsonianos e psicotrópicos }\end{array}$ & 36 & 7,0 \\
\hline
\end{tabular}


houve diferença importante no perfil das causas básicas de óbito para os anos comparados. A principal causa foi mantida, sendo estatisticamente significativa a diferença nas proporções registradas para os anos comparados $(\mathrm{p}<0,05)$.

Neste estudo, foi obtida uma maior proporção de óbitos no grupo etário de 20-39 anos $(44,0 \%)$ e $40-59$ anos (27,2\%). Os idosos ( $\geq 60$ anos) ficaram na $3^{\text {a }}$ posição em número de óbitos. Entre crianças com até 9 anos de idade, a faixa etária predominante foi de $\leq 4$ anos $(3,6 \%)$. A principal causa determinante no grupo etário de 20-39 anos foi a intoxicação de forma intencional/suicida $(54,9 \%)$. Isto também foi verificado nos grupos etários de 10-19 anos (52,7\%) e 4059 anos $(52,8 \%)$. A intoxicação acidental foi a principal causa determinante entre as crianças $\leq$ 4 anos (51,0\%). Quanto à população de idosos, a intoxicação com intenção indeterminada teve o maior registro de óbitos $(43,8 \%)$, seguida da suicida $(28,0 \%)$ e da acidental $(27,0 \%)$. As intoxicações do tipo acidental e homicida foram as que registraram óbitos em todos os grupos etários. No entanto, a forma intencional/suicida foi responsável por $2.125(48,3 \%)$ registros de óbitos por intoxicação com medicamentos.

Nos anos estudados, a taxa de mortalidade no Brasil por suicídio foi superior, em ambos os sexos, às outras circunstâncias de óbito. Nas intoxicações acidentais, as taxas de mortalidade para os homens foram maiores quando comparado com as das mulheres. Considerando o valor total das circunstâncias de óbito, a do tipo suicida foi a única que apresentou uma taxa de crescimento positiva (41\%), entre 1996 e 2005. Não houve diferenças importantes no padrão das taxas de mortalidade por circunstância de óbito e sexo (dados não apresentados). Cabe salientar que em $1.527(34,7 \%)$ registros a circunstância do óbito estava ignorada.

A Tabela 3 apresenta os APVP devido à mortalidade por intoxicação com medicamentos e, também, todas as causas de óbitos. Ao longo da década estudada, o impacto da mortalidade por esse tipo de agravo, medido em APVP, aumentou em $15,50 \%$ (TVAM $=1,61 \%$ ao ano) enquanto que para todas as causas de óbitos verificou-se uma variação negativa de $-11,85 \%$ (TVAM = $1,39 \%$ ao ano) no número de APVP. As idades, em ordem decrescente, que contribuíram com as maiores quantidades de perdas devido a esse agravo foram 27 (5.289), 19 (5.202) e 16 (5.076) anos. As intoxicações decorrentes de medicamentos apresentaram maior APVP por óbito em todos os anos estudados quando comparadas a todas as causas. Nesta década, registrou-se um acúmulo de 129.866 APVP por intoxicação com medicamentos (média $=12.987 \mathrm{APVP} / \mathrm{ano})$.

\section{Discussão}

Este estudo oferece um panorama farmacoepidemiológico da situação da mortalidade por intoxicação com medicamentos no Brasil a partir de dados do SIM/MS entre os anos de $1996 \mathrm{e}$

Tabela 3. Número de Anos Potenciais de Vida Perdidos devido à mortalidade por intoxicação com medicamentos e todas as causas. Brasil, 1996-2005.

\begin{tabular}{|c|c|c|c|c|}
\hline \multirow[b]{2}{*}{ Ano } & \multicolumn{2}{|c|}{ Intoxicação } & \multicolumn{2}{|c|}{ Todas as causas } \\
\hline & No APVP (\%) & APVP/óbito & No APVP & APVP/óbito \\
\hline & $14.185(0,08)$ & 32,4 & 16.978 .215 & 18,7 \\
\hline & $15.381(0,09)$ & 34,1 & 16.593 .368 & 18,4 \\
\hline $\begin{array}{l}1997 \\
1098\end{array}$ & $18.099(0,11)$ & 33,8 & 16.764 .584 & 18,0 \\
\hline $\begin{array}{l}1998 \\
1999\end{array}$ & $11.967(0,07)$ & 33,0 & 16.556 .703 & 17,6 \\
\hline $\begin{array}{l}1999 \\
2000\end{array}$ & $11.198(0,07)$ & 32,7 & 16.476 .389 & 17,4 \\
\hline 2001 & $14.259(0,09)$ & 33,9 & 16.104 .306 & 16,7 \\
\hline $\begin{array}{l}2001 \\
2002\end{array}$ & $14.094(0,09)$ & 33,2 & 16.031 .080 & 16,3 \\
\hline $\begin{array}{l}2002 \\
2003\end{array}$ & $15.960(0,10)$ & 37,5 & 15.940 .824 & 15,9 \\
\hline $\begin{array}{l}2003 \\
2004\end{array}$ & $14.723(0,09)$ & 33,5 & 15.723 .522 & 15,3 \\
\hline $\begin{array}{l}2004 \\
2005\end{array}$ & $16.384(0,11)$ & 32,0 & 14.966 .546 & 15,1 \\
\hline TC 96-05 & \multicolumn{2}{|c|}{$15,50 \%$} & \multicolumn{2}{|c|}{$-11,85 \%$} \\
\hline TVAM & \multicolumn{2}{|c|}{$1,61 \%$} & \multicolumn{2}{|c|}{$-1,39 \%$} \\
\hline
\end{tabular}

APVP= Anos Potenciais de Vida Perdidos; TC 96-05= Taxa de crescimento entre os anos 1996 e 2005; TVAM = Taxa de variação anual média 
2005. Este indicador de saúde pode ser um reflexo do padrão de consumo dos medicamentos no país atrelado à necessidade de aprimoramento das políticas de vigilância sanitária e também de fenômenos sociais como as desigualdades, a pobreza e o desemprego ${ }^{26}$.

As estatísticas sobre mortalidade constituemse um importante subsídio para o conhecimento do perfil epidemiológico de uma população, a elaboração de indicadores e o planejamento de ações de saúde. No Brasil, esses dados são rotineiramente sistematizados, desde 1975, no SIM/ MS, o mais antigo sistema de informação do Ministério da Saúde que identifica as principais causas de morte registradas no país.

O SIM/MS tem cobertura estimada de $82 \%$, com variação entre as regiões do país, principalmente no Norte e no Nordeste. Quanto à confiabilidade dos dados, está ocorrendo uma melhora gradativa no preenchimento de variáveis e, aproximadamente, $10 \%$ de óbitos são classificados como mal definidos. Outra limitação está relacionada com a taxa de mortalidade influenciada pelo sexo, idade e condições de vida da população ${ }^{21,27-30}$. Neste estudo, não houve a correção de sub-registros de óbitos nem a redistribuição das causas mal definidas, e a padronização da taxa de mortalidade foi realizada apenas por grupo etário.

Vários dos códigos da CID-10 selecionados não incluem os medicamentos como única substância responsável pelos óbitos. Além disso, um grupo farmacológico (classe de medicamentos) é citado, em muitos dos casos, de forma agregada com outros grupos. Diante dessas características não foi possível estabelecer qual a principal classe de medicamentos nem o princípio ativo envolvido com o agravo.

Um estudo com metodologia similar, realizado nos Estados Unidos da América em 2007, revelou que houve um aumento de $55 \%$ na mortalidade por intoxicação com medicamentos entre 1999 e $2003^{10}$. No Brasil, de 1996 a 2005, a taxa de crescimento foi de $17,8 \%$, destacando-se um aumento progressivo nos últimos cinco anos. Esta situação pode ser influenciada por fatores como a melhoria do acesso aos medicamentos, no processo de registro de óbitos (redução na subnotificação) e na definiçãa das causas mal definidas, bem como do aprimoramento da cultura da toxicovigilância e farmacovigilância no país.

Os resultados quanto à distribuição por sexo com predomínio masculino foram diferentes dos obtidos em uma pesquisa desenvolvida com a população cubana, na qual em praticamente to- dos os grupos de idades o sexo feminino foi preponderante ${ }^{2}$. No entanto, o suicídio, a principal circunstância do óbito, foi comum em ambas as populações ${ }^{2}$. Estudo publicado sobre o perfil dos óbitos de intoxicação registrados pelos CIAT brasileiros, entre 2002 e 2006, mostrou que houve uma maior concentração do sexo masculino e que o suicídio foi a circunstância mais prevalente ${ }^{15}$. Segundo Bochner e Souza ${ }^{15}$, as mulheres tentam mais contra a vida enquanto os homens obtêm mais sucesso em suas tentativas de suicídio.

As baixas taxas de mortalidade nas situações de homicídio encontradas estão condizentes com a literatura que cita, nesses casos, as armas de fogo como o principal meio de agressão ${ }^{31}$. O uso de medicamentos é mais comum nos casos de morte por suicídio. O grande percentual de intoxicação por medicamentos com intenção indeterminada pode ser atribuído ao medo das consequências, negligência ou vergonha de membros da família em revelar a verdadeira circunstância da intoxicação ${ }^{32}$. Para Hillman, apud Meneghel et al. ${ }^{33}$, existe sub-registro da morte por suicídio, o que varia de acordo com a região e a cultura, uma vez que o suicídio é um tabu na maioria dos grupos sociais.

Chama atenção, neste estudo, que nenhum código da CID-10 do Capítulo XIX (Lesões, envenenamento e algumas outras consequências de causas externas) foi encontrado como causa básica de óbito por intoxicação com medicamentos. Esta, é uma das informações mais importantes na Declaração de Óbito e que necessita atenção médica, pois o confundimento de causa terminal com a causa básica é um erro frequentemente cometido no preenchimento da Declaração ${ }^{34}$. Este problema pode produzir informações inadequadas relacionadas com essa variável.

Considerando a distribuição etária, observou-se uma maior concentração em adultos de 20 a 59 anos, cujo tipo de exposição foi intencional/suicida. Resultados semelhantes foram publicados com dados do SINITOX no período de 2002 a $2006^{15}$. A maioria das pessoas que morrem por intoxicação com medicamentos é adulta e os óbitos geralmente resultam de exposição intencional/suicida ${ }^{35}$.

Entre as crianças, a maioria das vítimas de óbitos foi de idade $\leq 4$ anos, sendo a principal causa determinante a intoxicação acidental. Estudo publicado por Matos e Nascimento ${ }^{12}$ mencionam as crianças desta faixa etária como as mais atingidas por intoxicação com medicamentos. Dos quatro anos em diante, as crianças compreendem melhor as orientações dos adultos e 
são mais seletivas quanto ao paladar. Ainda, segundo os autores, as cinco classes de medicamentos frequentemente mais envolvidas com óbito acidental em crianças $\leq 4$ anos são os descongestionantes nasais, os analgésicos, os broncodilatadores, os anticonvulsivantes e os contraceptivos orais ${ }^{12}$.

A maior frequência relativa da mortalidade por intoxicação com medicamentos foi registrada na região Sudeste. É nesta região onde se consome mais medicamentos e onde está quase metade das farmácias e drogarias oficialmente existente no país ${ }^{12}$. Resultados publicados com dados do SINITOX mostram que na região Centro-Oeste foi registrada a maior taxa de letalidade para os medicamentos ${ }^{15}$. Neste estudo, a partir de 2002, na região Centro-Oeste foram registradas as maiores taxas padronizadas de mortalidade do país.

Ao analisar a variável "anos de estudo" (proxy da renda) e sua relação com a intoxicação com medicamentos foi verificado que os resultados entre os grupos de comparações não foram estatisticamente significativos. No entanto, as pessoas com $\leq 3$ anos de estudo representaram aproximadamente metade dos óbitos por intoxicação com medicamentos. Uma das hipóteses para explicar este achado é que, nos últimos dez anos, o acesso da população brasileira de baixa renda aos medicamentos foi ampliado.

As intoxicações com medicamentos resultaram em uma importante causa de óbito prematura, resultando em perda social e econômica para a sociedade. Os APVP indicam uma grandeza dos custos indiretos impostos por determinado agravo, cuja mensuração não implica na valorização monetária da vida humana ${ }^{36}$. Entretanto, como previamente descrito, são os adultos em fase laboral ativa que mais frequentemente morrem por intoxicação com medicamentos. Neste caso, a morte prematura desses indivíduos gera impacto econômico negativo na sociedade.

Além dos registros hospitalares e do SINITOX, fica evidente a importância do uso potencial do SIM/MS como fonte de informação em farmacoepidemiologia com a finalidade de subsidiar o aprimoramento de políticas de vigilância sanitária. Ademais, sugere-se a realização de um estudo comparativo entre os dados do SIM/MS e SINITOX para avaliar a exatidão dos dados de mortalidade disponíveis no país. Uma via possível para melhorar a informação sobre mortalidade por intoxicação com medicamentos seria o estabelecimento de parcerias entre os CIAT e os codificadores de causas de morte das secretarias municipais de saúde.

\section{Colaboradores}

DM Mota foi responsável pelo planejamento e execução do estudo e pela redação do manuscrito. JRR Melo, DRC de Freitas e M Machado participaram do planejamento do estudo e revisão final do texto.

\section{Agradecimentos}

À Coordenação do Programa de Treinamento em Epidemiologia Aplicada aos Serviços do SUS da Secretaria de Vigilância em Saúde do Ministério da Saúde (EPISUS/SVS/MS).

\section{Referências}

1. Brasil. Lei no 5.991 , de 17 de dezembro de 1973. Dispõe sobre o controle sanitário do comércio de drogas, medicamentos, insumos farmacêuticos e correlatos, e dá outras providências. Diário Oficial da União 1973; $21 \mathrm{dez}$.

2. Valiente MLG, Echmendia JOP, Delgado CAG, Marrero BC. Mortalidad por intoxicaciones agudas producidas con medicamentos. Cuba, 1995-1996. Rev Cubana Farm 2000; 34(1):23-33.

3. Bortoleto ME, Bochner R. Impacto dos medicamentos nas intoxicações humanas no Brasil. Cad Saude Publica 1999; 154(4):859-869.

4. Mendonça RT, Marinho JL. Discussão sobre intoxicações por medicamentos e agrotóxicos no Brasil de 1999 a 2002. Rev Eletr de Farm 2005; 2(2):45-63.

5. Mena CH, Bettini MS, Cerda PJ, Concha F, Paris EM. Epidemiología de las intoxicaciones en Chile: una década de registros. Rev Méd Chile 2004; 132(4): 493-499.

6. Portugal. Instituto Nacional de Emergência Médica. Relatório anual de actividades, 2007. Centro de Informação Antivenenos - CIAV: 21. [Documento na Internet] 2007. [acessado 2008 nov]. Disponível em: http://www.inem.pt/files/2/documentos/201004211 80040349052.pdf 
7. Watson WA, Litovitz TL, Rodgers GC, KleinSchwartz W, Reid N, Youniss J, Flanagan A, Wruk KM. 2004 Annual Report of the American Association of Poison Control Centers Toxic Exposure Surveillance System. 2005: p. 589-666. [acessado 2008 jul 28]. Disponível em: http://www.poison.org/prevent/documents

8. Lambert H, Manel J, Bellow A, Kouch S. Morbidity and mortality from acute drug poisoning in France. Rev Prat 1997; 47(7):716-720.

9. Caballero PJ, Dorado MS, Jerez B, Medina M, Brusint B. Vigilancia epidemiológica de la intoxicación aguda en el Área Sur de la Comunidad de Madrid. Ana de Med Intern 2004; 21(2):62-68.

10. Wysowski DK. Surveillance of prescription drugrelated mortality using death certificate data. Drug Safety 2007; 30(6):533-540.

11. Moghadamnia AA, Abdollahi M. An epidemiolog ical study of poisoning in northern Islamic Republic of Iran. East Mediterr Health J 2002; 8(1):88-94.

12. Matos GC, Nascimento AC. Impacto dos medicamentos como agentes de intoxicações humanas. Revista Racine 2008; 106(5):59-66.

13. Arrais PSD, Coelho HLL, Batista MCDS, Carvalho ML, Righi RE, Arnau JM. Perfil da automedicação no Brasil. Rev Saude Publica 1997; 31(1):71-77.

14. Rozenfeld S. O uso de medicamentos no Brasil. In: Laporte JR, Tognoni G, Rozenfeld S, organizadores. Epidemiologia dos medicamentos: princípios gerais. São Paulo: Hucitec; 1989. p.21-41.

15. Bochner R, Souza VMFA. Panorama das intoxicações e envenenamentos registrados no Brasil pelo Sistema Nacional de Informações Tóxico-Farmacológicas (SINITOX). Revista Racine 2008; 106(5):4458.

16. Rozenfeld S. Agravos provocados por medicamentos em hospitais do Estado do Rio de Janeiro, Brasil. Rev Saude Publica 2007; 41(1):108-115.

17. Paes NA. Avaliação da cobertura dos registros de óbitos dos Estados brasileiros em 2000. Rev Saude Publica 2005; 39(6):882-890.

18. Margonato FB, Thomson Z, Paoliello MMB. De terminantes nas intoxicações medicamentosas agudas na zona urbana de um município do Sul do Brasil. Cad Saude Publica 2008; 24(2):333-341.

19. Gandolfi E, Andrade MGG. Eventos toxicológicos relacionados a medicamentos no Estado de São Paulo. Rev Saude Publica 2006; 40(6):1056-1064.

20. Gomes FBC. Sistema de Informações sobre Mortalidade: Considerações sobre a qualidade dos dados. Informe Epidemiológico do SUS 2002; 11(2):5-6

21. Laurenti R, Jorge MHPM, Gotlieb SLD. A confiabilidade dos dados de mortalidade e morbidade por doenças crônicas não-transmissíveis. Cien Saude Colet 2004; 9(4):909-920.

22. Brasil. Ministério da Saúde (MS). Departamento de Informática. DATASUS. Informações em saúde: mortalidade. [acessado 2008 nov]. Disponível em: http:/ /tabnet.datasus.gov.br/tabdata/sim/dados/cid10 indice.htm

23. Organização Mundial da Saúde (OMS). Classificação Estatística Internacional de Doenças e Problemas Relacionados à Saúde. 10a Revisão. São Paulo: Edusp; 1995.
24. Instituto Brasileiro de Geografia e Estatística (IBGE). Informações de saúde. Notas técnicas. Banco de dados do Sistema Único de Saúde (DATASUS). [acessado 2008 nov]. Disponível em: http://tabnet. datasus.gov.br/cgi/deftohtm.exe?ibge/cnv/popuf. defdoDATASUS

25. World Health Organization (WHO). Age standardization of rates: a new who standard. GPE Discus sion Paper Series no 31. Geneva: World Health Organization (WHO); 2001.

26. Souza ER, Lima MLC, Veiga JPC. Impacto da violência na saúde dos brasileiros. Brasília: Ministério da Saúde (MS); 2005.

27. Prado RR, Nascimento, AF, Souza, MFM. Brasil 2006 - Uma análise da desigualdade em saúde. Brasília: Ministério da Saúde (MS); 2007.

28. Sasson D, Silveira DP, Santos IS, Machado JP, Souza SMM, Mendes S. Saúde Brasil 2006 - Uma análise da desigualdade em saúde. Brasília: Ministério da Saúde (MS); 2007.

29. Cordeiro LD, Alencar AP, Rocha FMM, Nascimento A F, Stevens A, Souza, MFM, Bierrenbach ALS. Mortalidade infantil no Brasil: determinantes e desigualdade. In: Brasil. Ministério da Saúde (MS). Secretaria de Vigilância em Saúde. Departamento de Análise de Situação em Saúde, organizador. Saúde Brasil 2006 - Uma Análise da Desigualdade em Saúde. $1^{\text {a }}$ ed. Brasília: Editora Ministério da Saúde; 2006. p. 249-304

30. Organização Pan-Americana da Saúde (OPAS). In dicadores básicos para a saúde no Brasil: conceitos e aplicações. 2a ed. Brasília: Organização Pan-Americana da Saúde (OPAS); 2008.

31. Conceição GMS, Soares-Filho AM, Ramalho WM, Montenegro MMS, Morais-Neto OL. Saúde Brasi 2006 - Uma análise da desigualdade em saúde. Brasília: Ministério da Saúde (MS); 2007.

32. Gawryszewski VP, Koizumi MS, Mello-Jorge MHP As causas externas no Brasil no ano de 2000: comparando a mortalidade e a morbidade. Cad Saude Publica 2004; 20(4):995-1003.

33. Meneghel SN, Victora CG, Faria NMX, Carvalho LA, Falk JW. Epidemiological aspects of suicide in Rio Grande do Sul, Brasil. Rev Saude Publica 2004; $38(6): 1-6$

34. Pedrosa LDCO, Sarinho SW, Ordonha MR. Análise da qualidade de informação sobre causa básica de óbitos neonatais registrados no Sistema de Informação sobre Mortalidade: um estudo para Maceió, Alagoas, Brasil, 2001-2002. Cad Saude Publica 2007; 23(10):2385-2395.

35. Goodman \& Gilman. As bases farmacológicas da terapêutica. $10^{\text {a }}$ ed. Rio de Janeiro: McGraw-Hill; 2005

36. Iunes RF. Impacto econômico das causas externas no Brasil: um esforço de mensuração. Rev Saude Publica 1997; 31(Supl. 4):38-46.

Artigo apresentado em 14/10/2009

Aprovado em 30/11/2009

Versão final apresentada em 10/12/2009 